\title{
A compact fluorescence and polarization near-field scanning optical microscope
}

\author{
G. Merritt, E. Monson, E. Betzig, and R. Kopelman ${ }^{\text {a) }}$ \\ Department of Chemistry, University of Michigan, 930 N. University Avenue, Ann Arbor, Michigan 48109
}

(Received 10 October 1997; accepted for publication 22 April 1998)

\begin{abstract}
We present a transmission, fluorescence, and polarization near-field scanning optical microscope with shear-force feedback control that is small in size and simple to operate. This microscope features an ultrafine mechanical tip/sample approach with continuous manual submicron control over a range of several millimeters. The piezo-driven $12 \mu \mathrm{m} x-y$ scan range is complimented by a $4 \mathrm{~mm}$ coarse mechanical translation range in each direction. The construction materials used in the mechanical feedback loop have been carefully chosen for thermal compatibility in order to reduce differential expansion and contraction between the tip and sample. A unique pressure-fit sample mount allows for quick and reliable sample exchange. Shear-force feedback light is delivered to the scan head via an optical fiber so that a remote laser of any type may be used as a source. This dither light is collimated and refocused onto the tip, delivering a consistently small spot which is collected by a high numerical aperture objective. This new scan head incorporates an optical system which will permit the linearization of scan piezo response similar to a scheme used successfully with atomic force microscopy. This is designed to both overcome the piezo's inherent hysteresis and to eliminate drift during long duration spatial scans or spectroscopic measurements at a single location. The scan head design offers added flexibility due to the use of optical fibers to deliver the dither and scan linearization light, and functions in any orientation for use in conjunction with upright or inverted optical microscopes. (C) 1998 American Institute of Physics. [S0034-6748(98)02707-5]
\end{abstract}

\section{INTRODUCTION}

Resolution in optical microscopy has traditionally been limited by the nature of far-field optics, as Abbé ${ }^{1}$ showed more than a century ago: the best obtainable resolution is on the order of half of the wavelength of the light used. A way to circumvent this barrier was suggested by Synge ${ }^{2}$ in 1928 : by exiting an aperture of subwavelength dimensions, light near the aperture is confined to approximately the dimensions of that aperture. This principle was first demonstrated in the microwave regime ${ }^{3}$ in 1972, but only became practical at optical wavelengths ${ }^{4-8}$ when tapered, subwavelength aperture optical probes were designed for use with scanned probe microscope techniques [such as those developed with the scanning tunneling microscope $(\mathrm{STM})^{9}$ and the atomic force microscope (AFM)]. ${ }^{10}$ Combined with shear-force feedback $^{11,12}$ to maintain a small separation between the tip aperture and the sample, near-field scanning optical microscopy (NSOM) allows the user to routinely achieve resolution that is independent of Abbé's diffraction limit.

Several critical performance requirements of the typical shear-force feedback controlled transmission/fluorescence near-field microscope place severe restrictions on microscope design requirements (Fig. 1). While other scanning microscopies such as STM typically favor a scanning probe and stationary sample, most near-field detection schemes favor a nontranslating tip since the remote detection devices do not have to track a signal which shifts in space. Such a con-

\footnotetext{
${ }^{a)}$ Electronic mail: kopelman@umich.edu
}

figuration also naturally lends itself to conventional laserbased shear-force feedback techniques that are well established and relatively simple to implement. Most NSOM designs therefore delegate the scanning duties to the sample, which is driven by a piezoelectric transducer. Since sensitive transmission/fluorescence NSOM requires that high numerical aperture optics be placed very close to the near-field aperture, the designer must generally leave exposed the halfplane of space on the side of the sample opposite the tip. Unlike typical AFM designs, the piezoelectric transducer that scans the sample must lie on the same side of the sample as the probe. This nested design with the probe (and its dither piezo) placed inside of a four-quadrant sample piezo tube would be easier to execute if a sample piezo of large diameter were used. Unfortunately, scan range decreases with increasing tube diameter. Increasing the length of the scan tube would recover lost scan range, but increase the dimensions of the tip/sample mechanical feedback loop. A physically larger mechanical feedback loop has a lower resonance frequency, which translates into poorer shear-force feedback response times.

Here we describe a near-field/shear-force microscope (Fig. 2) that addresses these conflicting design requirements yet remains flexible, reliable, and easy to operate. This microscope is also quite compact (the main body is a cylinder approximately $5 \mathrm{~cm}$ in diameter and $7.5 \mathrm{~cm}$ in length) and has been designed to operate in conjunction with upright or inverted optical microscopes, or in any arbitrary orientation that a given application may require. The use of optical fibers to deliver light to the shear-force detection system and $X-Y$ 


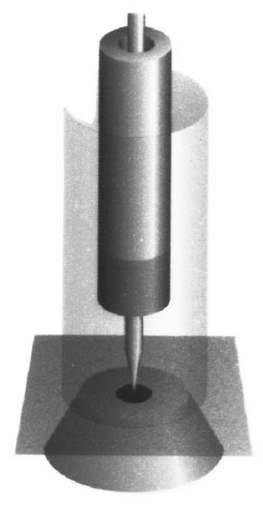

FIG. 1. Schematic of the typical near-field scanning optical microscope, showing the spatial relationship of the major components. At the center is the tapered near-field fiber probe. Moving outward, the next cylinder represents the dither piezo (nearest the tip) and its supporting element. This is surrounded by the cylindrical piezo that generates the sample's raster scan. An optical microscope objective collects light from beneath the sample. Since the objective occupies the space directly below the sample, all other major scan head elements must lie in the half-plane above the sample in a compact design.

scan linearization system allows a wide variety of supporting light sources to be used-virtually anything that can be coupled into an optical fiber. Furthermore, while this microscope is not intended for low-temperature use, it served as a design study for a low-temperature NSOM; many of its design features (including its small volume and the materials used in its construction) reflect this.

\section{MECHANICAL}

We designed this microscope in cylindrically symmetric layers, with each layer dedicated to a separate task (Fig. 3). At the center is the optical fiber near-field tip glued inside of a 1-mm-diam borosillicate or quartz capillary tube. In studies for which auto-fluorescence from the fiber jacket or glass capillary is significant, a second metal capillary (steel tube stock intended for hypodermic needle use) can be glued into the glass capillary before the fiber is inserted. To further reduce fluorescence background from the tip, opaque silver print is used instead of organic or polymeric adhesive (e.g., cyanoacrylate or epoxy). The glass or quartz capillary is held in place at the center of a small cylindrical piezoelectric

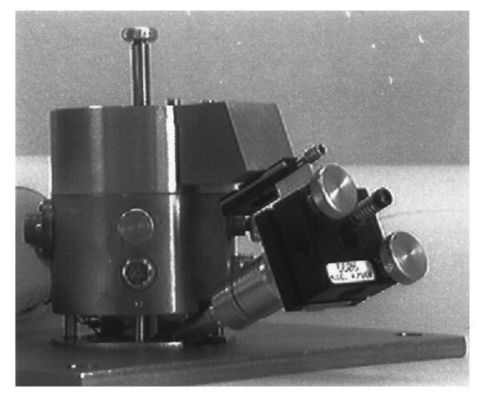

FIG. 2. Near-field scanning optical microscope. Cylindrical main body diameter is $\sim 5 \mathrm{~cm}$. Dither laser optics package, translator, and mirror mount assembly are at right.

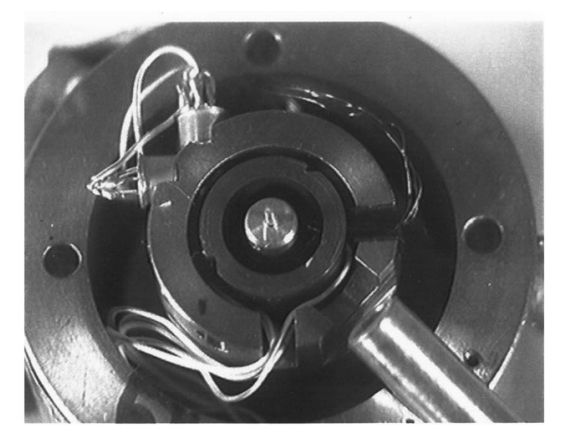

FIG. 3. Mechanical layout. Moving outward from the center are the following elements: (a) a near-field fiber probe in quartz capillary; (b) tip holder, (c) sample raster scan piezo with sample holder anchor (sample/sample holder not shown); (d) $X-Y$ position optics for scan linearization; (e) microscope body and coarse positioning elements. Dither light optics are at lower right. Position sensitive detectors for scan linearization are at upper left. Image width is $\sim 50 \mathrm{~mm}$.

transducer (3/16 in. diam $\times 3 / 16$ in. length, 0.020 in. wall thickness) that is used to dither the tip for shear-force feedback detection.

Moving outward, the next layer consists of the raster scan piezo $(1 / 2$ in. diam $\times 1$ in. length, 0.020 in. wall thickness) and the sample holder mount. Surrounding the scan piezo is a cylinder containing the detectors that monitor the fine positioning of the sample. The outermost layer, the microscope body, contains the coarse positioning elements.

\section{A. Coarse positioning}

The coarse positioning tasks for both tip approach (" $Z$ ", positioning) and sample region selection (" $X-Y$ " positioning) are carried out by systems of fine pitch screws and return springs. While these two kinds of motions are carried out independently, many components of the microscope play a dual role since they are involved in both vertical (tip) and lateral (sample) adjustments.

The near-field tip rides on the $Z$ translation slug (Fig. 4). The slug is mounted on 80 turns per inch screws which rotate in place as they are turned, for they are secured to the microscope body with caged ball bearings, not via their threads. As the screws turn, the $Z$ translation slug advances or retracts along the screws depending on the direction of screw rotation. The three 1/4-80 screws are driven in unison by a $4: 1$ reduction gear drive, giving an effective pitch of 320 turns per inch so that one complete revolution by hand of the external tip approach knob generates a tip advance of $\sim 83 \mu \mathrm{m}$. This is equivalent to one micron of tip advance for a rotation of $4^{\circ}$; given a $Z$ offset range of $\sim 2.5 \mu \mathrm{m}$ in the scan piezo, the sample can be approached easily and rapidly by hand without crashing the tip.

The sample assembly is attached to the $Z$ translation slug with extension springs (Fig. 4). The extension springs hold the sample assembly rigidly against tungsten carbide ball bearings at the end of the $z$ translation screws; the bearings turn against sapphire disks on the sample assembly. As the $Z$ translation screws turn to advance the tip, the sample assembly is not displaced. The tension on the extension springs is sufficient to hold the sample assembly firmly in place through the entire range of motion of the $Z$ translation slug. 


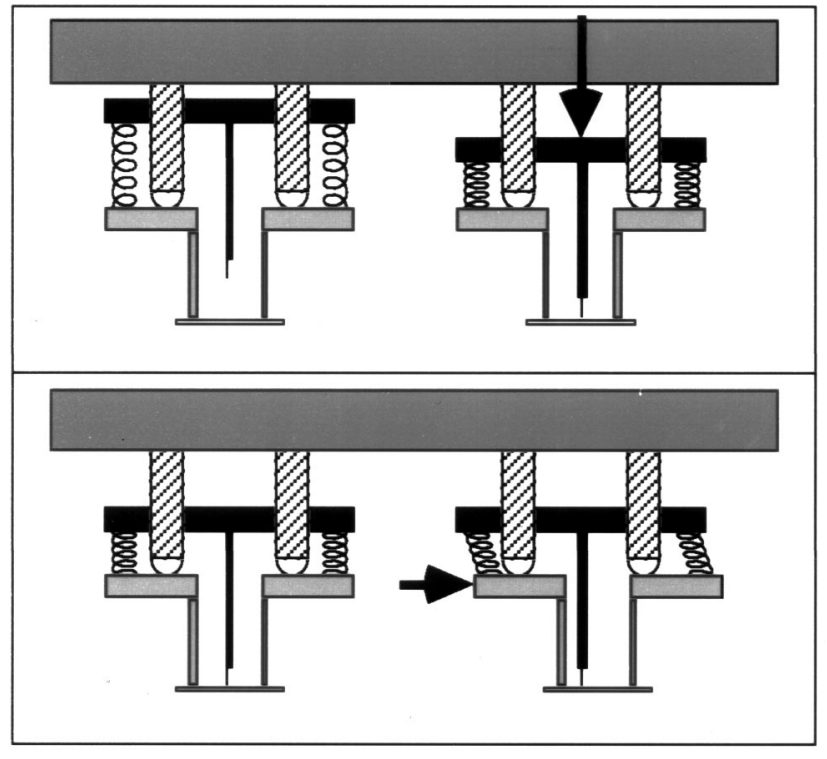

FIG. 4. Top: Coarse $Z$ translation. As the $Z$ translation screws turn in place, the $Z$ translation slug (which holds the near-field tip) advances or retracts. Arrow indicates tip advance. Extension springs hold the sample assembly in place. The figure shows two screw and spring sets for clarity; actual microscope features three pairs spaced $120^{\circ}$ apart. Bottom: Coarse $X-Y$ positioning (one dimension shown). Sample assembly is pushed in each dimension by a fine pitch screw as indicated by arrow. Return compression spring lies opposite screw (not shown) in each dimension.

While the sample assembly is constrained to be motionless in the vertical $(Z)$ direction, it can be moved freely in the $X-Y$ plane (Fig. 4). These lateral adjustments allow scan access to a $16 \mathrm{~mm}^{2}$ area of the sample. Given the scan piezo's maximum scan area of $144 \mu \mathrm{m}^{2}$, there are more than $10^{5}$ distinct $12 \mu \mathrm{m} \times 12 \mu \mathrm{m}$ scan regions accessible by the user simply by turning the external coarse adjusting screws.

\section{B. Shear-force feedback detection}

The tip's dither motion is detected by focusing laser light onto the side of the fiber and using lock-in detection of the scattered light to monitor the shear-force signal, similar to the technique described in Ref. 13. The laser light is delivered to the side of the near-field probe by an optical fiber (separate from the near-field fiber), allowing virtually any laser to be used as a source; a $3 \mathrm{~mW}$ diode laser emitting at $780 \mathrm{~nm}$ is used in this application. This optical fiber is mounted to a two component lens system (Fig. 5). The first lens, a Thor Labs aspheric collimation optic with antireflec-

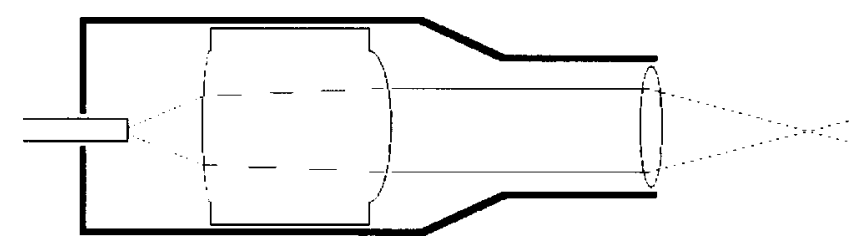

FIG. 5. Optics for shear-force feedback detection system. A dedicated single-mode optical fiber delivers $780 \mathrm{~nm}$ light to a custom-made lens package. Light from the fiber is collimated by an aspheric collimation optic and focused to a spot by a second lens. The entire package is affixed to a mirror mount, which in turn is mounted to a linear translator that moves the package along the optical axis. These three degrees of freedom allow the focused spot to be positioned on the taper of the near-field tip.
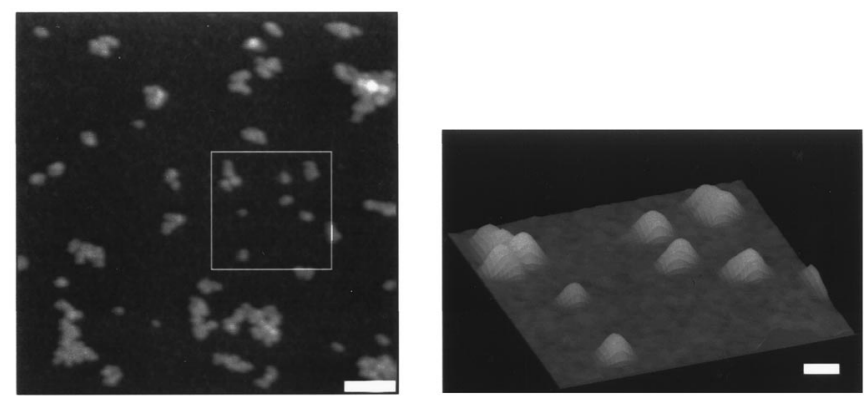

FIG. 6. Left: Topographic (shear-force feedback) image of small aggregates of 19-nm-diam latex microspheres. Microspheres can be individually identified, and noise in the direction normal to the sample is approximately 1 to $2 \mathrm{~nm}$. Scan size is $1.5 \mu \mathrm{m} \times 1.5 \mu \mathrm{m}$; scale bar is $200 \mathrm{~nm}$. Right: 3D rendition of outlined area in first image. Scale bar is $50 \mathrm{~nm}$. Effective shear-force tip size is $<50 \mathrm{~nm}$.

tive coating, collimates the light exiting the fiber; the second lens focuses the beam. The fiber and lens assembly is mounted as a unit onto a mirror mount with two degrees of freedom which allow the optical axis of the assembly to be aimed at the tip. The mirror mount is in turn mounted to a linear positioner. These three adjustment motions allow the focused laser spot to be positioned anywhere along the dithering fiber's taper and easily compensate for the slightly different mounting location of every tip (Figs. 2 and 3).

Light scattered from the dithering tip is collected by the same high numerical aperture (NA) objective that collects the near-field signal. The $780 \mathrm{~nm}$ emission wavelength of the shear-force laser source is easily separated from the shorter wavelength near-field transmission and fluorescence signal with a short pass filter. The shear-force light is imaged onto a photomultiplier tube (PMT). The PMT signal is sent to a lock-in amplifier by way of a current to voltage converter and a divider circuit that corrects for intensity variations due to laser fluctuations or changes in sample transmittivity. The lock-in amplifier compares the amplitude and phase of this corrected signal to the sine wave that drives the dither piezo. The signal used for feedback is the lock-in's $X=R \cos \phi$, where $R$ is the amplitude of the component of the PMT signal at the driving (reference) frequency, and $\phi$ is the relative phase between the input and reference signals. The shear-force feedback interaction is predominantly due to damping, and manifests itself in changes in $R$ and $\phi$. A feedback circuit modulates the $Z$ position of the sample piezo so that a constant tip/sample interaction is maintained, keeping the tip within a few nanometers of the sample while scanning.

The coarse approach of the tip is monitored by viewing the tip through the sample with the same inverted optical microscope that collects the near-field signal. When the tip is within a few microns of the sample, the final approach is made by alternately advancing the tip and engaging the shear-force feedback. We typically operate the shear-force feedback with a dither frequency of 30 to $100 \mathrm{kHz}$ and a tip dither amplitude of $\sim 5 \mathrm{~nm}$. This is typically achieved with several millivolts ac applied to the dither piezo. The shearforce feedback topographic image in Fig. 6 demonstrates the performance of this system. 


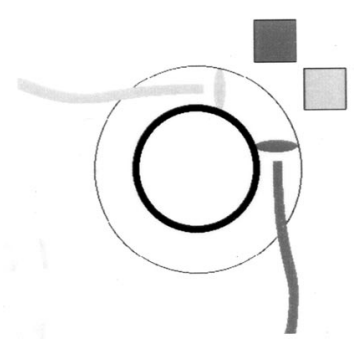

FIG. 7. Schematic diagram of scan piezo monitoring system. Optical fibers deliver light to 2.2-mm-diam lenses affixed to sample holder anchor. Light from fibers is imaged on position sensitive detectors.

The original design for the dither detection optics was similar to that described above, but the focusing lens was fixed to the microscope body. Orienting the mirror positioner so that the focused spot was incident on the tip often required that the laser light hit the lens off axis, producing an unsatisfactory focus. The current configuration allows for much more precise operation, as an optimized focus is maintained through all adjustment positions.

\section{Sample and tip mounting}

The sample mounting system for this microscope was designed to allow the user to quickly and easily exchange samples while providing a mechanical connection that is solid enough to maintain the integrity of the mechanical feedback loop. The tight fit is achieved by a 1/16-in.-diam Delrin ${ }^{14}$ sphere in the sample holder anchor which protrudes slightly into the hole that receives the sample holder. When the sample holder is inserted into the anchor the Delrin ball is compressed, securing the sample holder. Samples are prepared on No. 0 glass cover slips that are $\sim 100 \mu \mathrm{m}$ thick and $12 \mathrm{~mm}$ in diameter. The cover glass is glued to the sample stage with cyanoacrylate adhesive ("super glue") applied around the entire circumference. The large contact area between sample and holder provides extremely rigid support of the sample. The cover slips are typically destroyed when they are removed from the sample stage, but the use of many interchangeable sample stages allows for flexibility of use.

The tip holder was originally designed with the same Delrin ball configuration as the sample holder anchor, but variations in the external diameters of the quartz capillary tubes used to hold the fiber tips made this technique unreliable. The Delrin ball in the tip holder was thus replaced by a 0-80 set screw which secures the capillary in the tip holder.

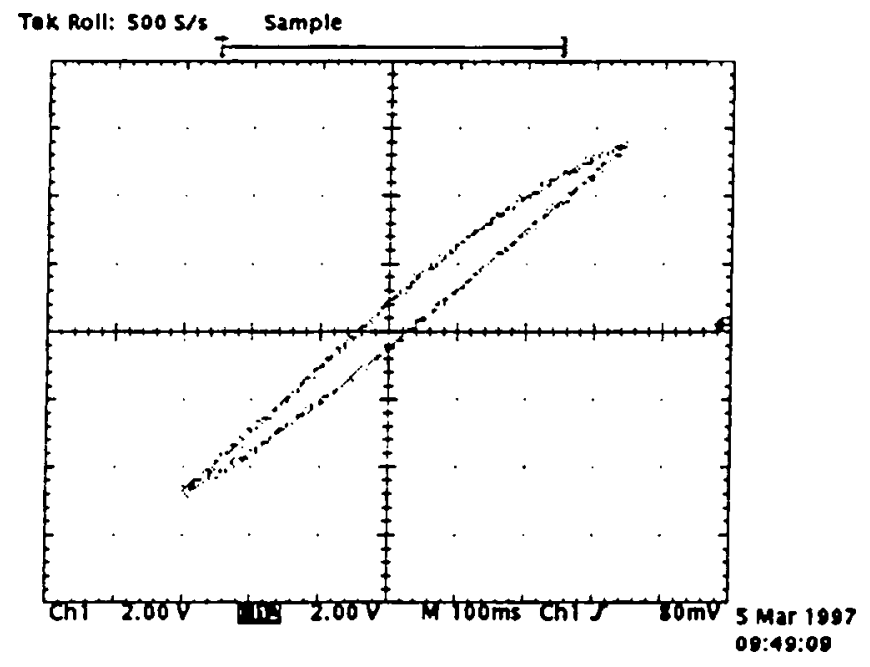

FIG. 8. Oscilloscope $X-Y$ plot of position sensitive detector signal vs piezo driving voltage. Nonlinear response shows piezo hysteresis.

\section{Scan linearization}

Since piezoelectric scan tubes are subject to hysteresis, nonlinear response to changes in control voltages, and drifting over time while control voltages are held constant, it is desirable to eliminate these effects via position monitoring and feedback control. This microscope incorporates a system for linearizing the response of the sample scan piezo that is similar to a system used successfully with AFM. ${ }^{15}$ Built into this near-field microscope are two identical optical monitoring systems, one for each of the orthogonal scan directions of the sample piezo (Figs. 3 and 7). These signals must be routed into a separate feedback circuit that corrects the scan control voltages as necessary to achieve a linear response. For each direction a 2.2-mm-diam lens is mounted in the sample holder anchor, and therefore translates as the sample piezo generates a raster scan. An optical fiber (independent of the near-field tip and the shear-force detection light source) delivers laser light to one side of the lens; the fiber is imaged by the lens onto a position sensitive detector. As the lens shifts with piezo movements, the light from the optical fiber shifts across the face of the position sensitive detector. Figure 8 shows an example of the response of the optical detection system. The slight asymmetry of the curve probably indicates a small detection nonlinearity or positional offset, but the overwhelming hysteresis could only come from the piezo motion. This signal also showed the expected trends of increased hysteresis with increasing scan speed and

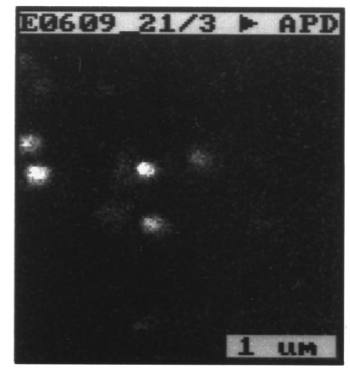

(a)

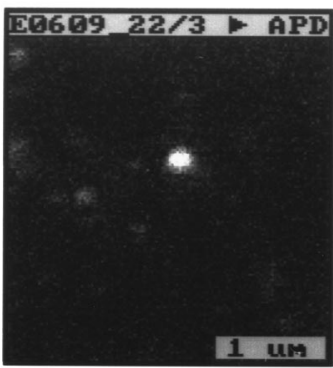

(b)

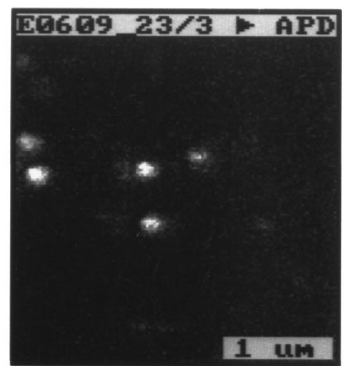

(c)
FIG. 9. Near-field fluorescence images of single DiI molecules on PMMA. The same $3 \times 3$ micron area was scanned three times $(128 \times 128$ pixels, $10 \mathrm{~ms}$ pixel). Between scans (a) and (b) the tip output polarization was rotated $+90^{\circ}$, which caused changes in observed fluorescence from each molecule since each has a different excitation dipole orientation. Between scans (b) and (c) the tip output polarization was rotated $-90^{\circ}$ to return to the original orientation; scan (c) matches (a) as expected. 

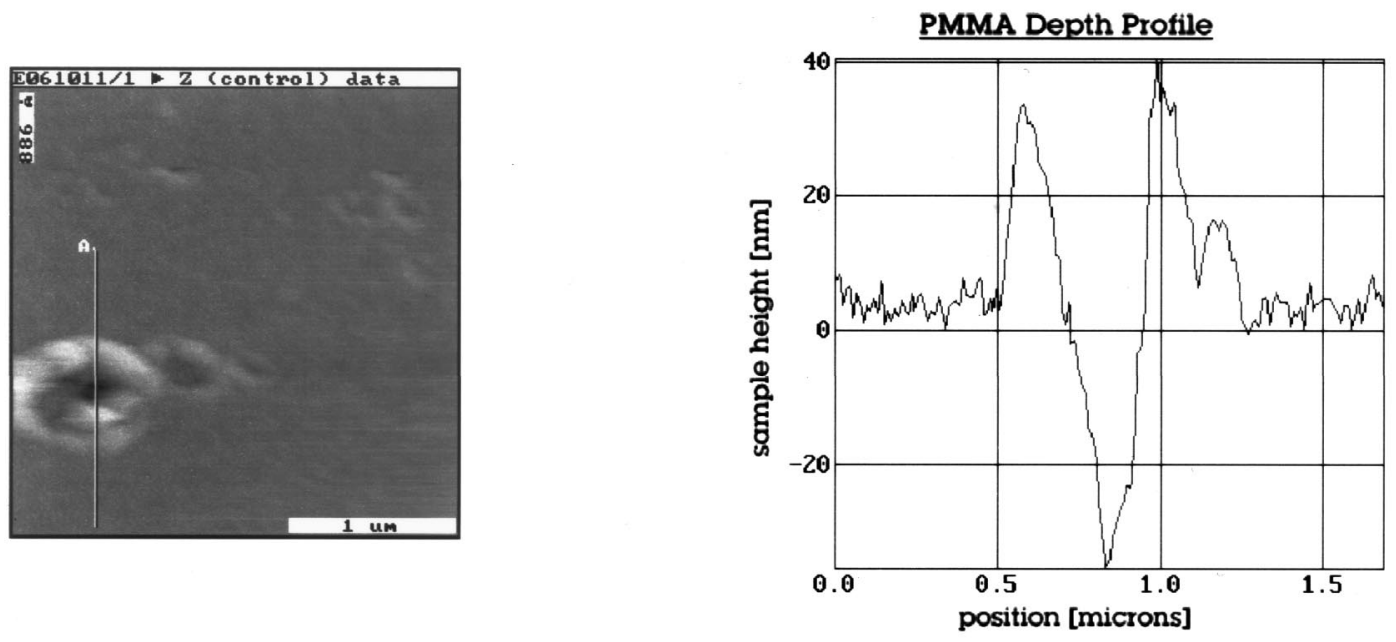

FIG. 10. Shear-force feedback data from the sample described in Fig. 9. Left: The microscope tip was driven through the PMMA to the glass substrate on a uniformly flat region of the sample to create impressions. Right: A line scan sampled from the image shows the thickness of the PMMA film to be 25-30 nm.

amplitude. Final evaluation of the system will only come through implementation of the complete feedback system.

\section{E. Materials}

As discussed above, this microscope was created both to be a high-performance room temperature near-field microscope and as a design study for an as yet unbuilt low temperature microscope. Material selection criteria therefore included not only a consideration of mechanical properties appropriate for the tip/sample mechanical feedback loop, but also thermal expansion properties over a wide range of temperatures. The tip and sample piezos have thermal expansion coefficients of $\sim 3 \times 10^{-6} \mathrm{~mm} / \mathrm{mm} / \mathrm{K}$. Titanium is used extensively in the mechanical feedback loop; its thermal expansion coefficient is $9 \times 10^{-6} \mathrm{~mm} / \mathrm{mm} / \mathrm{K}$, at least a factor of two lower than typical varieties of steel, aluminum, and brass. Since the piezos cannot be attached to a conductor, the insulating composite Vespel is used sparingly for piezo mounting. While its thermal expansion coefficient is $\sim 50$ $\times 10^{-6} \mathrm{~mm} / \mathrm{mm} / \mathrm{K}$, it offers superior machinability to close tolerances when compared to other available insulators.

The $Z$ translation screws are tipped with tungsten carbide ball bearings that turn against sapphire disks on the sample assembly (Fig. 4). Previous experience with similar designs indicated that this junction between the $Z$ translation screws and the sample assembly demanded materials of high stiffness (for a high mechanical resonance of the tip/sample feedback loop) and low friction (for smoothness of operation). Stainless steel, brass, and aluminum were used for the remaining components.

\section{OPERATION}

The authors operate this instrument on an Olympus IX-70 infinity corrected inverted microscope optimized for fluorescence imaging, although the scan head has been designed to operate in any orientation and in conjunction with a wide variety of collection optics. A short-pass filter below the objective angled at $45^{\circ}$ to the optical axis directs the 780 $\mathrm{nm}$ shear-force feedback detection light out of the rear of the microscope, where it is imaged onto a photomultiplier tube. The near-field excitation light (488 or $514 \mathrm{~nm}$, from an argon-ion laser) is rejected from the optical path during fluorescence imaging by a holographic notch filter, and a bandpass filter centered about the emission peak of the fluorophores rejects additional excitation and shear-force detection light. The coarse approach of the tip is monitored by viewing the tip through the sample with the same inverted optical microscope that collects the near-field signal and a video camera on the optical microscope is used to align the shearforce detection system. When the tip is within a few microns of the sample, the final approach is made by alternately engaging the shear-force feedback and advancing the tip. The piezo raster scan and shear-force feedback are controlled by RHK Technology, Inc. electronics in conjunction with RHK software running on a 486 computer.

\section{RESULTS}

The authors have used this microscope successfully for fluorescence imaging of single molecules, including the effects of variations in excitation polarization. Sample preparation was accomplished by first cleaning coverslips in a solution of concentrated sulfuric acid and hydrogen peroxide for ten minutes, then rinsing thoroughly with double distilled water and oven drying. Poly(methylmethacrylate) (PMMA) films were spin coated at $7000 \mathrm{rpm}$ for $25 \mathrm{~s}$ from a $1 \%$ solution in chlorobenzene, which should result in films of about $20 \mathrm{~nm}$ thickness. ${ }^{16} \operatorname{DiI}\left(C_{18}\right)$ (molecular probes) was then applied to the sample as $10 \mu \ell$ of a less than $1 \mathrm{nM}$ solution in methanol and allowed to air dry. Samples were then stored in darkness for several days before scanning. Figure 9 shows three sequential images of single DiI molecules on a PMMA film $(128 \times 128$ pixels, $10 \mathrm{~ms} /$ pixel $)$. This series illustrates not only the polarization preservation of our tips, but more importantly the sensitivity and stability of our system.

Shear-force feedback was used to further characterize this sample by performing a depth profile of the PMMA film (Fig. 10). The near-field tip was driven into a uniformly flat 
area of the sample, and the dimpled area was subsequently scanned in shear-force mode to map the resulting topography. The depth of the PMMA was measured to be in the range of 25-30 nm. This information allows us to modify the sample preparation recipe to obtain the desired thickness for future studies.

\section{ACKNOWLEDGMENTS}

This work was supported by NSF Grant No. DMR9410709 and by the University of Michigan, Office of Vice President for Research.

${ }^{1}$ E. Abbé, Arch. Mikrosc. Anat. Entwicklungsmech. 9, 413 (1873).

${ }^{2}$ E. H. Synge, Philos. Mag. 6, 356 (1928).

${ }^{3}$ E. A. Ash and G. Nicholls, Nature (London) 237, 510 (1972).

${ }^{4}$ U. Ch. Fischer and H. P. Zingsheim, J. Vac. Sci. Technol. 19, 881 (1981).
${ }^{5}$ A. Lewis, M. Isaacson, A. Muray, and A. Harootunian, Biophys. J. 41, 405a (1983).

${ }^{6}$ A. Lewis, M. Isaacson, A. Harootunian, and A. Muray, Ultramicroscopy 13, 227 (1984).

${ }^{7}$ D. W. Pohl, W. Denk, and M. Lanz, Appl. Phys. Lett. 44, 651 (1984).

${ }^{8}$ E. Betzig, J. K. Trautman, T. D. Harris, J. S. Weiner, and R. L. Kostelak, Science 251, 1468 (1991).

${ }^{9}$ G. Binnig, H. Rohrer, Ch. Gerber, and E. Weibel, Phys. Rev. Lett. 49, 57 (1982).

${ }^{10}$ G. Binnig, C. F. Quate, and C. Gerber, Phys. Rev. Lett. 56, 930 (1986).

${ }^{11}$ R. Toledo-Crow, P. C. Yang, Y. Chen, and M. Vaez-Iravani, Appl. Phys. Lett. 60, 2957 (1992).

${ }^{12}$ E. Betzig, P. L. Finn, and J. S. Weiner, Appl. Phys. Lett. 60, 2484 (1992).

${ }^{13}$ F. F. Froehlich and T. D. Milster, Appl. Phys. Lett. 65, 2254 (1994).

${ }^{14}$ Delrin is an acetal resin made by DuPont. Its relevant characteristics in this application include high strength, impact resistance and fatigue endurance, and inherent lubricity.

${ }^{15}$ R. C. Barrett and C. F. Quate, Rev. Sci. Instrum. 62, 1393 (1991).

${ }^{16}$ D. E. Bornside, C. W. Macosko, and L. E. Scriven, J. Electrochem. Soc. 138, 317 (1991) 